\title{
Novel association of severe neonatal encephalopathy and Hirschsprung disease in a male with a duplication at the $\mathrm{Xq} 28$ region
}

\author{
Raquel M Fernández ${ }^{1,2}$, Rocío Núñez-Torres ${ }^{1,2}$, Antonio González-Meneses ${ }^{3}$, Guillermo Antiñolo ${ }^{1,2}$, \\ Salud Borrego ${ }^{1,2^{*}}$
}

\begin{abstract}
Background: Hirschsprung disease (HSCR) is a neurocristopathy characterized by the absence of parasympathetic intrinsic ganglion cells in the submucosal and myenteric plexuses along a variable portion of the intestinal tract. In approximately $18 \%$ of the cases HSCR also presents with multiple congenital anomalies including recognized syndromes.

Methods: A combination of MLPA and microarray data analysis have been undertaken to refine a duplication at the Xq28 region.

Results: In this study we present a new clinical association of severe neonatal encephalopathy (Lubs syndrome) and HSCR, in a male patient carrying a duplication at the Xq28 region which encompasses the MECP2 and L1CAM genes.

Conclusions: While the encephalopathy has been traditionally attributed to the MECP2 gene duplication in patients with Lubs syndrome, here we propose that the enteric phenotype in our patient might be due to the dosage variation of the LICAM protein, together with additional molecular events not identified yet. This would be in agreement with the hypothesis previously forwarded that mutations in L1CAM may be involved in HSCR development in association with a predisposing genetic background.
\end{abstract}

\section{Background}

Hirschsprung disease (HSCR, OMIM 142623) is a congenital malformation of the hindgut characterised by the absence of parasympathetic intrinsic ganglion cells in the submucosal and myenteric plexuses. It is regarded as the consequence of the premature arrest of the craniocaudal migration of vagal neural crest cells in the hindgut between the fifth and $12^{\text {th }}$ week of gestation to form the enteric nervous system (ENS) and is therefore regarded as a neurocristopathy [1]. HSCR occurs as an isolated trait in $70 \%$ of cases. Genetic mapping in families and mutational screening of candidate genes, together with the study of several natural and knockout animal models, clearly have shown the involvement of

\footnotetext{
* Correspondence: salud.borrego.sspa@juntadeandalucia.es

'Unidad de Gestión Clínica de Genética, Reproducción y Medicina Fetal, Instituto de Biomedicina de Sevilla (IBIS), Hospital Universitario Virgen del Rocío/CSIC/Universidad de Sevilla, Sevilla, Spain

Full list of author information is available at the end of the article
}

multiple genes in the pathogenesis of HSCR [1,2]. Of them, RET proto-oncogene is the major gene involved in the disease, with classical coding hypomorphic mutations accounting for $50 \%$ of familial cases and $7-35 \%$ of sporadic patients [2], and a common variant within a transcriptional enhancer of its intron 1 which seems to have a prominent role for a great proportion of sporadic cases [3].

On the other hand, a chromosomal abnormality is associated in $12 \%$ of HSCR cases, trisomy 21 being by far the most frequent (>90\%). Associated congenital anomalies are found in $18 \%$ of the HSCR patients. Among those HSCR-associated syndromes, there exist some clinical presentations with central nervous system anomalies, including the HSAS/MASA spectrum (OMIM 307000 and 303350) ascribed to mutations in the X-linked $L 1 C A M$ gene $[1,4]$. Indeed, until now mutations of the L1CAM gene (OMIM 308840) have 
been found in nine out of ten patients reported to show association of X-linked hydrocephalus with HSCR [4-10]. In addition, two cases with both acrocallosal syndrome and HSCR have been also linked to L1CAM mutations [11]. Therefore, although the question of L1CAM being a modifier gene in HSCR has been raised with no definitive answer given thus far $[7,8]$, the whole data suggest that mutations in L1CAM may be involved in HSCR development in association with a predisposing background [9].

In this report we present a clinical case of neonatal severe encephalopathy (OMIM 300673) associated with HSCR, presenting with a duplication in the X chromosome encompassing the MECP2 (OMIM 300005) and L1CAM genes among others. To our knowledge this is the first time in which this kind of association has been described and an extensive analysis has been performed in order to completely dissect its molecular cause.

\section{Methods}

\section{Patients}

The affected patient was the second child born to a healthy couple. He was born at full term with a birth weight of $3.500 \mathrm{Kg}$. He was hypotonic and failed to thrive in the neonatal period. The patient presented with severe constipation, a distended abdomen, and intestinal biopsy confirmed Hirschsprung disease with aganglionosis extending up to the sigmoid. During his first years of life he developed tonic-clonic seizures and frequent respiratory tract infections. MRI scan showed a discrete increase of the subarachnoid space and dilatation of the ventricular system, cavum septum pellucidum and cavum vergae. He was clinically diagnosed with neonatal encephalopathy, benign external hydrocephalus and sideropenia. The child is currently 13 years old and has not developed speech nor walked, although he can sit without support. He is dysmorphic with a long thin face, down slanting palpebral fissures, divergent strabismus, prognatism, marked ojival palate, long fingers and bilateral cryptorchid. He also presents bilateral hip dislocation. Molecular testing for Angelman and Rett syndrome was negative, and karyotype was normal.

As shown in the family tree (Figure 1), our index patient had a cousin who shared some of his clinical signs and died at the age of 1 , fifteen years ago. Clinical reports for this patient describe a congenital midline malformation, with unspecified ventricular alterations and partial agenesis of the corpus callosum. Unfortunately no additional clinical information is available for this patient.

We had also available the DNA from the mother, sister, three maternal aunts and two maternal cousins of our index patient (individuals II.1, II.3, II.5, II.6, III.2, III.4 and III.6 of the family tree). An informed consent was obtained from all the participants for clinical and molecular genetic studies. The study conformed to the tenets of the declaration of Helsinki and was approved by the "Committee of Ethics and Clinical Investigation" from Hospitales Universitarios Virgen del Rocío.

\section{MLPA analysis}

The MLPA (Multiple Ligation Probe-dependent Amplification) method employed was as described by Schouten et al. [12], using MLPA kits purchased from MRC-Holland (Amsterdam, The Netherlands), and according to manufacturer's recommendations. MLPA is a variation of the PCR that permits multiple targets to be amplified with only a single primer pair. Each probe consists of a two oligonucleotides which recognise adjacent target sites on the DNA. One probe oligonucleotide contains the sequence recognized by the forward primer, the other the sequence recognised by the reverse primer.

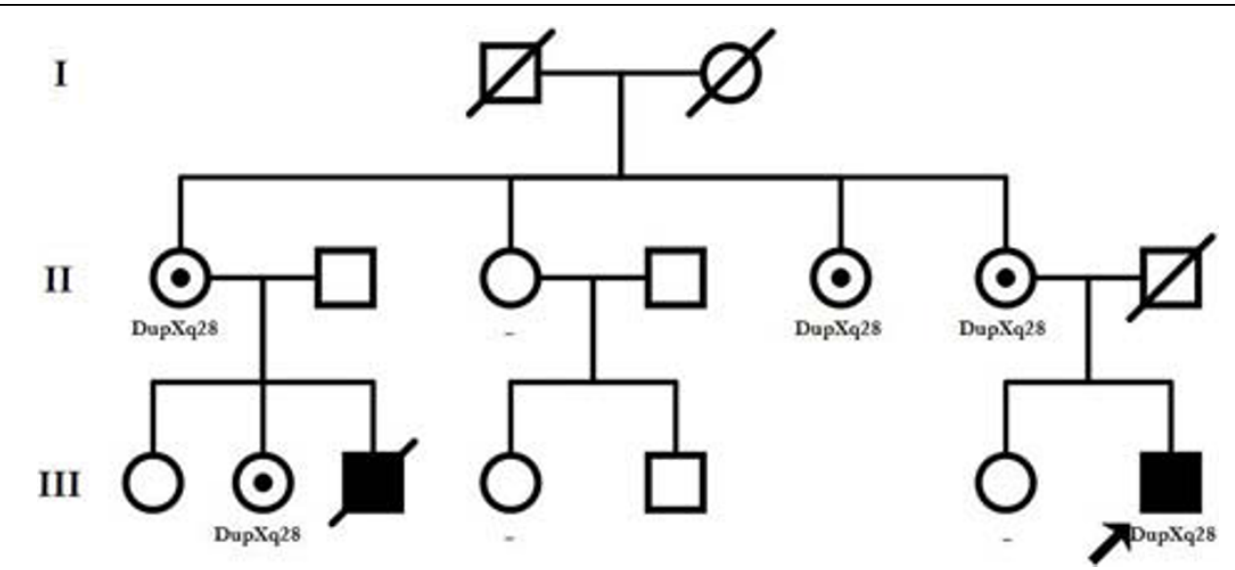

Figure 1 Pedigree of the patient carrying the duplication at Xq28. Together with the index patient (III.7), individuals II.1, II.5, II.6 and III.2 were found to be healthy carriers of the rearrangement, while II.3, III.4 and III.6 resulted negative for the testing. 
Only when both probe oligonucleotides are hybridized to their respective targets, can they be ligated into a complete probe. Each complete probe has a unique length, so that its resulting amplicons can be separated and identified by capillary electrophoresis. Since the forward primer used for probe amplification is fluorescently labeled, each amplicon generates a fluorescent peak which can be detected by a capillary sequencer. Comparing the peak pattern obtained on a given sample with that obtained on various reference samples, the relative quantity of each amplicon can be determined. Initial MLPA studies were aimed to completely rule out the possibility of any microdeletion syndrome included in the P245 kit. When a duplication was observed for the three probes binding the MECP2 gene, MLPA was performed using the P015D kit, which included probes for the four exons of this gene, together with IRAK1, L1CAM, IDH3G and SLC6A8, alongside $12 \mathrm{X}$ chromosome and four autosomal control probes. The P106 kit for X-linked mental retardation syndromes, comprising 3 genes at $\mathrm{Xq} 28$ (GDI1, AFF2, SLC6A8) and several other genes along the whole $\mathrm{X}$ chromosome was also employed to analyze the extent of the duplication. Subsequently and in order to verify the results, we used the P049 kit which covered a series of genes at Xq.28 as FMR2, IDS, PNCK, SLC6A8, BCAP31, ABCD1, IDH3G, L1CAM, IRAK1, MECP2, FLNA, GDI1, FVIII and $S Y B L 1$, and also included six chromosome $\mathrm{X}$ control probes. Fragment analyses were performed using the 3730 DNA analyzer (Applied Biosystems, Foster City, CA, USA) and for data analysis we used GeneMarker v 1.6 (Softgenetics L.L.C). We normalized the samples by the peak height, and included control individuals who had previously been confirmed to have no CNVs of the studied genes. Furthermore, the assays were performed in duplicated to confirm the robustness of the analysis.

\section{Microarray analysis}

DNA samples from our patient and his mother were hybridized with Genome wide human SNP 6.0 arrays (Affymetrix), in order to analyze the presence CNVs. Samples $(20 \mu \mathrm{l})$ diluted to $50 \mathrm{ng} / \mu \mathrm{l}$ were processed in the GeneChip Instrument System platform (Affymetrix UK L) following the manufacturer's recommendations. Copy number data were generated by comparing intensities for both SNP and copy number probes "in silico" to the HapMap control provided by Affymetrix. The resulting $\log 2$ ratios were then analysed using a Hidden Markov Model (HMM) to generate copy number calls for each probe. The quality of the $\log 2$ data was assessed by the degree of variation, determined by the MAPD metric. MAPD is defined as the Median of the Absolute value of all Pairwise Differences between log2 ratios for a given chip. High MAPD > 0.4 (using the
HapMap control) is considered to be the cut-off at which copy numbers can no longer be accurately called.

\section{$\mathrm{X}$ inactivation analysis}

$\mathrm{X}$ inactivation assays performed for the female subjects of the family as well as for controls, consisted in the examination of the human androgen-receptor gene (HUMARA, Xq11.2) methylation status, as described by Allen et al. [13]. This study was pertinent to explain the complete absence of any clinical manifestation in all the female carriers of the rearrangement within this family. Samples were separated on an ABI3730 automated DNA sequencer (Applied Biosystems) and were analyzed with GeneMapper software for peak positions and area intensity calculations. These data were further processed with the use of Excel.

\section{Mutational screening, SNPs genotyping and haplotyping}

PCR - dHPLC analysis was undertaken for the mutational screening of RET and other HSCR related genes such as EDNRB, EDN3, SOX10, PHOX2B, GDNF and $N T N$, using previously described conditions [14,15]. The whole coding sequence of the L1CAM gene was also screened in both the affected child and his mother by direct sequencing (conditions available on request).

RET SNPs c.73-126G > T (rs2565206), c.73-1370C > T (rs2505532), c.73-1463T > C (rs2505533) and c.73+ 9277T > C (rs2435357) were genotyped in our patient employing Taqman based techniques for allelic discrimination (TaqMan ${ }^{\circledR}$ SNP Genotyping Assay, Applied Biosystems, Foster City, CA) as previously described [16].

\section{Results}

Investigation of the affected patient using the different MLPA kits demonstrated a duplication at Xq28 including MECP2, IRAK1, L1CAM, IDH3G and a region of $A B C D 1$ from exon 3 upstream. Normal dosages were observed for exons 1 and 2 of $A B C D 1$ as well as for the remaining neighbouring genes GDI1, SLC6A8, BCAP31 or PNCK. Taking into account the relative positions of all these genes, the duplication seemed to comprise approximately a region of a minimum of $297 \mathrm{~Kb}$. Subsequently, by performing copy number analysis using the Affymetrix 6.0 SNP chip, we were able to confirm the presence of the rearrangement and characterized it in detail (Figure 2). As shown in figure 3, a duplication encompassing $621 \mathrm{~Kb}$ and containing two stretches of $65,8 \mathrm{~Kb}$ and $35.1 \mathrm{~Kb}$ of non-duplicated sequences, was detected in our index patient.

Segregation analysis of the family by MLPA showed that the duplication was not a de novo event but had been inherited from his mother, and also was carried by other four healthy females within the family, including the mother of the deceased cousin (Figure 1). X inactivation 


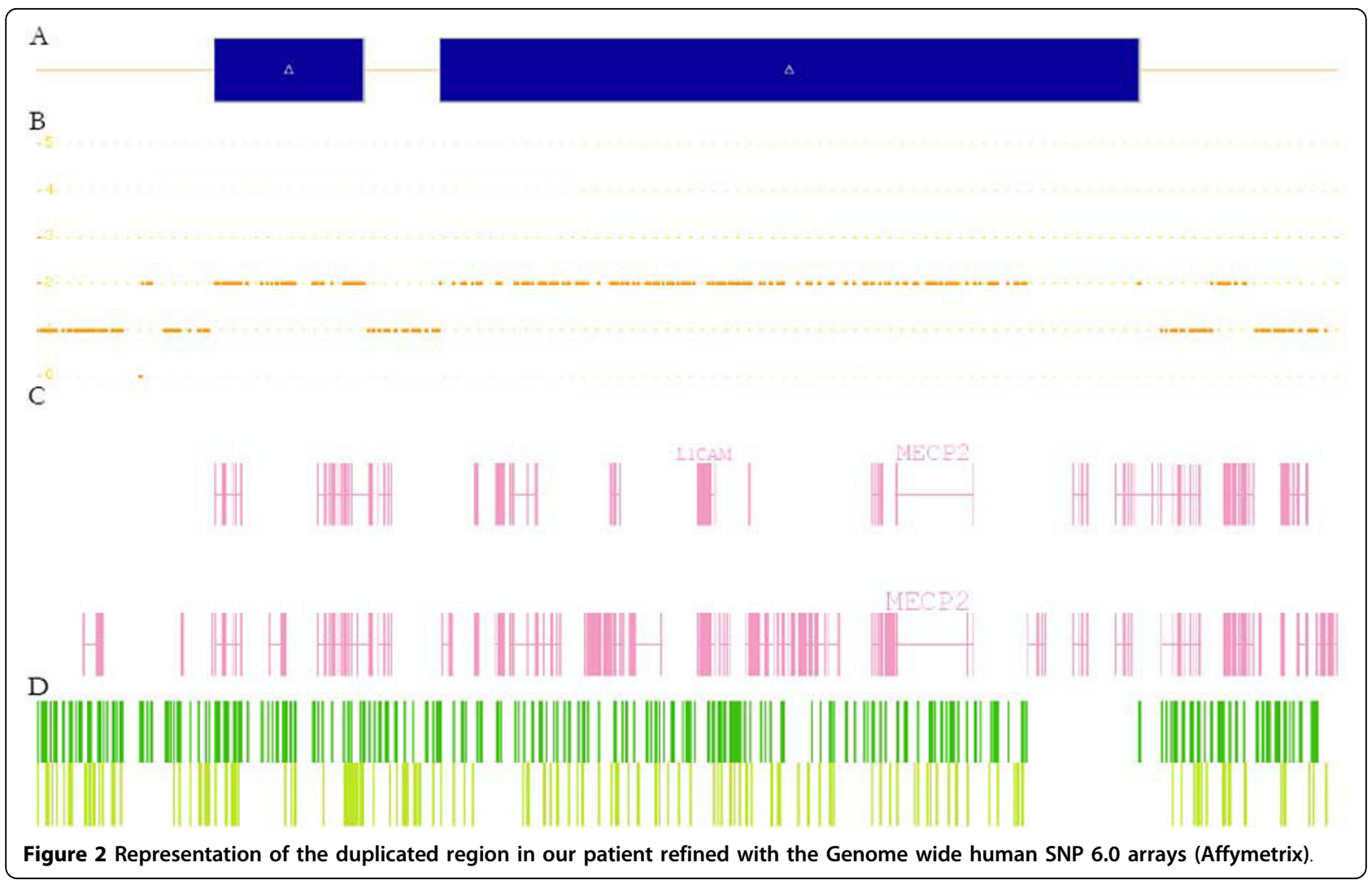

testing on the DNA samples for all the asymptomatic female carriers, showed a marked skewed inactivation with the inactive $\mathrm{X}$ chromosome being the duplicated one $(99,999975 \%$ of active normal allele versus $0,000025 \%$ of active mutant allele), which would clearly explain the absence of any clinical feature in those individuals.

On the other hand, the mutational screening of the coding regions of $R E T$ and the remaining genes tested including L1CAM, revealed no mutations which could be attributable to HSCR phenotype in our patient. Moreover molecular testing of the RET haplotype comprising specific variants within its intron 1 , showed that this patient did not carry the "HSCR-associated combination" $[17,18]$, nor the so-called "enhancer mutation" [3].

\section{Discussion}

Over 50 patients have to date been reported with Xq28 duplications varying in size from $0.2 \mathrm{Mb}$ to larger scale

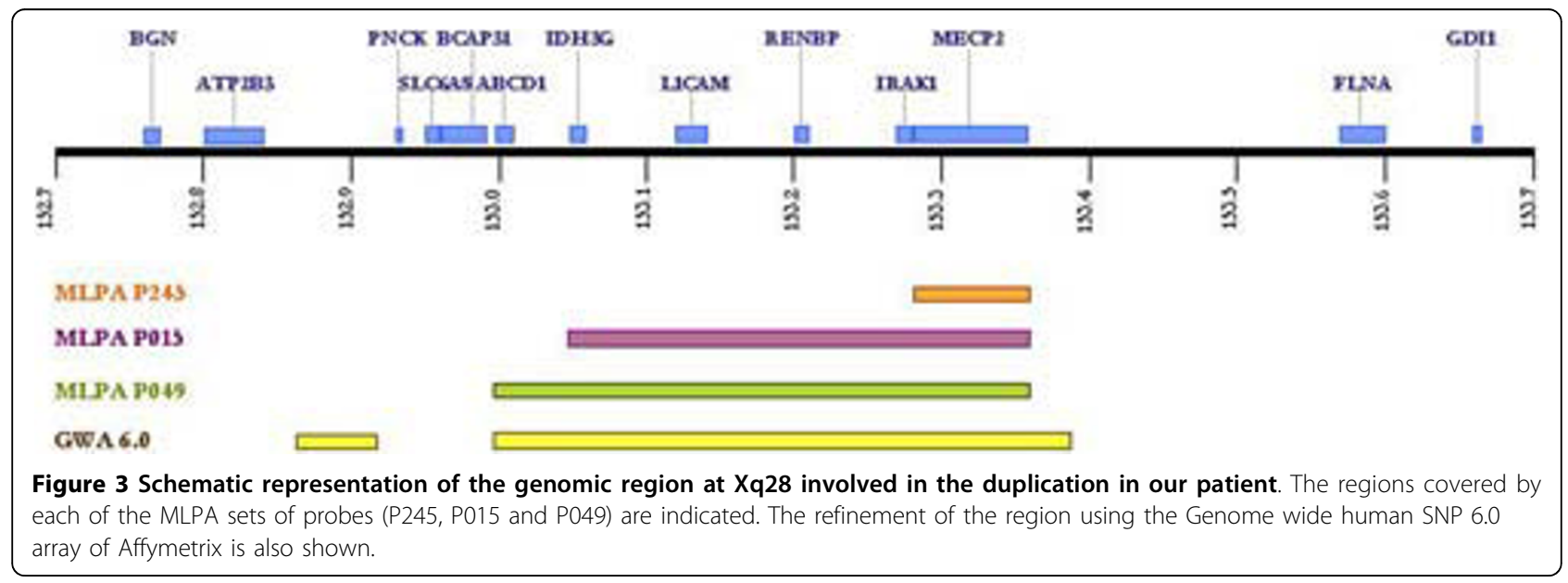


duplications extending beyond Xq27. Most have included the $M E C P 2$ and $L 1 C A M$ genes, although none of them have been reported to present with Hirschsprung disease [19-21]. It has been suggested that the clinical features associated to neonatal encephalopathy in males carrying the $\mathrm{Xq} 28$ duplication might be solely due to the $M E C P 2$ gene, as patients with duplications involving this region alone have been reported with the same classical phenotype [19,21]. Indeed, an OMIM number is used to specifically design the disorder caused by duplication or triplication of MECP2 (OMIM\#300260; Lubs X-Linked mental retardation syndrome). Although spasticity, severe learning disability, axial hypotonia and frequent chest infections have been common findings within this group of patients, gastrointestinal symptoms have been much rarer. Only worth of note, in the report by Clayton-Smith et al., eight families with several male affected patients carrying Xq28 duplications had presented with intestinal pseudo-obstruction or bladder distension, but these features were attributed exclusively to the duplication of the FLNA gene [21]. Several arguments supported such assumption, since these authors had also found 2 other families carrying intragenic duplications of FLNA and presenting exclusively the enteric phenotype. Moreover, Gargiulo et al. had already reported point FLNA mutations in X-linked families with pseudo-obstruction [22]. The pathogenic mechanism proposed by the authors to explain how both duplications and truncating mutations of the FLNA gene could lead to intestinal pseudo-obstruction, was the hypothesis that the amount of FLNA would be critical for neuronal migration, and either its increased or decreased expression could interfere with the normal migration process [21]. In our particular case we could propose exactly the same pathogenic mechanism for the connection between the L1CAM gene and Hirschsprung disease in our patient carrying the duplication at Xq28. The L1 cell adhesion molecule is a membrane glycoprotein belonging to a large class of immunoglobulin superfamily cell adhesion molecules (CAMs) that mediate cell-to-cell adhesion at the cell surface. The L1CAM protein is found primarily in the nervous system and is important in neuronal adhesion, migration, neurite outgrowth, and myelination [4]. Mutations in the L1CAM gene cause neurological abnormalities of variable severity, including congenital hydrocephalus, agenesis of the corpus callosum, spastic paraplegia, bilaterally adducted thumbs, aphasia, and mental retardation (see OMIM). Interestingly, $L 1 C A M$ is the only gene included in the duplicated region which has been verified to be a ENSexpressed gene and to play a key role in its development during embryogenesis [23], and some cases of patients with either X-linked hydrocephalus or acrocallosal syndrome and HSCR have been reported to present
L1CAM mutations (visit HGMD http://www.hgmd.cf.ac. uk/ac/all.php). In contrast, none of the remaining genes encompassed by the rearrangement, such as IRAK1 or IDH3G have been previously related to HSCR. All the L1CAM mutational events reported so far to be responsible for the manifestation of the corresponding clinical pictures, are point sequence changes $[4,6,8,10,11]$ or small deletions [5,9], whose proposed pathogenic mechanisms lead to the suspicion that decreased L1CAM may be a modifying factor in the development of HSCR [4]. It would be licit to speculate that also an increase in the amount of L1CAM, due to a duplication in the dosage of this gene, might be involved someway in the pathogenesis of Hirschsprung in this particular patient. In other words it would be plausible that the amount of L1CAM would be critical during ENS development, and either an increase or decrease of this protein could interfere with the processes of neuronal adhesion and migration.

The association between encephalopathy and HSCR should not be considered surprising as brain development is largely controlled by the same neural growth factors acting in the ENS [24]. Because the frequency of Lubs X-linked mental retardation syndrome is estimated to be extremely low (only around 50 cases being reported to date), and the frequency of HSCR is approximately 1:5000, then the anticipated incidence of both together would be almost inexistent by chance alone. So it is not probable that the association of both clinical phenotypes has occurred accidentally and by different molecular events. This statement is supported by the fact that L1CAM abnormality has been previously proposed to contribute to HSCR onset $[8,9,25]$. Parisi et al. hypothesized that in those cases in which X-linked hydrocephalus presents together with HSCR, either RET or another HSCR gene contributes to aganglionosis under the influence of a defective L1CAM gene, and L1CAM may act as an $\mathrm{X}$ linked modifier gene for the development of HSCR [8]. We therefore propose a similar role for L1CAM in the context of the association of Lubs syndrome and HSCR. Given the complex nature of HSCR, it would be conceivable that the duplication at $\mathrm{Xq} 28$ region including the L1CAM gene, together with another still unidentified molecular events, could lead to the manifestation of the disease in our patient. The existence of those additional molecular events would also explain why no other patients carrying MECP2-L1CAM duplications reported to date present with HSCR [19-21]. In addition, we have also to argue that children with congenital severe neonatal encephalopathy cannot communicate symptoms such as constipation, and originally, they have a constipation tendency; so there may be several cases of HSCR among those patients that goes undiagnosed because of a premature 
death, just as it occurred with the cousin of our index patient.

\section{Conclusions}

Although a more comprehensive analysis of additional HSCR genes is warranted, our results support an involvement of L1CAM duplication in the pathogenesis of HSCR, and present evidence of a possible novel syndromic form of Hirschsprung disease.

\section{Acknowledgements}

We would like to thank the family that participated in this study. This study was funded by Fondo de Investigación Sanitaria, Spain (PI070080, and PI071315 for the E-Rare project), Consejeria de Innovación Ciencia y Empresa de la Junta de Andalucia (CTS 2570), and Consejeria de Salud de la Junta de Andalucia (PI0249-2008). The CIBER de Enfermedades Raras is an initiative of the ISCIII.

\section{Author details}

'Unidad de Gestión Clínica de Genética, Reproducción y Medicina Fetal, Instituto de Biomedicina de Sevilla (IBIS), Hospital Universitario Virgen del Rocío/CSIC/Universidad de Sevilla, Sevilla, Spain. ${ }^{2}$ Centro de Investigación Biomédica en Red de Enfermedades Raras (CIBERER), Sevilla, Spain. ${ }^{3}$ Unidad de Gestión Clínica de Pediatría, Hospital Universitario Virgen del Rocío, Avda Manuel Siurot s/n, 41013, Sevilla, Spain

\section{Authors' contributions}

RMF and RN-T carried out the molecular genetic studies. AG-M delineated the clinical presentation of the index patient and recruited the family. SB coordinated and supervised all the analyses. RMF and SB drafted the manuscript, and GA collaborated with valuable contributions to the manuscript. All authors have read and approved the final manuscript.

\section{Competing interests}

The authors declare that they have no competing interests.

Received: 8 April 2010 Accepted: 22 September 2010 Published: 22 September 2010

\section{References}

1. Amiel J, Sproat-Emison E, Garcia-Barcelo M, Lantieri F, Burzynski G, Borrego S, Pelet A, Arnold S, Miao X, Griseri P, Brooks AS, Antinolo G, de Pontual L, Clement-Ziza M, Munnich A, Kashuk C, West K, Wong KK, Lyonnet S, Chakravarti A, Tam PK, Ceccherini I, Hofstra RM, Fernandez R, Hirschsprung Disease Consortium: Hirschsprung disease, associated syndromes and genetics: a review. J Med Genet 2008, 45:1-14.

2. Chakravarti A, Lyonnet S: Hirschsprung Disease. In The metabolic and molecular bases of inherited disease. Edited by: Scriver CS. McGraw-Hill: 2002:6231-6255, 2002.

3. Emison ES, McCallion AS, Kashuk CS, Bush RT, Grice E, Lin S, Portnoy ME, Cutler DJ, Green ED, Chakravarti A: A common sex-dependent mutation in a RET enhancer underlies Hirschsprung disease risk. Nature 2005, 434:857-63.

4. Okamoto N, Del Maestro R, Valero R, Monros E, Poo P, Kanemura Y, Yamasaki M: Hydrocephalus and Hirschsprung's disease with a mutation of L1CAM. J Hum Genet 2004, 49:334-337.

5. Okamoto N, Wada Y, Goto M: Hydrocephalus and Hirschsprung's disease in a patient with a mutation of L1CAM. J Med Genet 1997, 34:670-671.

6. Vits L, Chitayat D, Van Camp G, Holden JJ, Fransen E, Willems PJ: Evidence for somatic and germline mosaicism in CRASH syndrome. Hum Mutat 1998, , Suppl 1: S284-S287.

7. Hofstra RM, Elfferich P, Osinga J, Verlind E, Fransen E, Lopez Pison J, de DieSmulders CE, Stolte-Dijkstra I, Buys CH: Hirschsprung disease and L1CAM: is the disturbed sex ratio caused by L1CAM mutations? J Med Genet 2002, 39:E11.
8. Parisi MA, Kapur RP, Neilson I, Hofstra RM, Holloway LW, Michaelis RC, Leppig KA: Hydrocephalus and intestinal aganglionosis: is L1CAM a modifier gene in Hirschsprung disease? Am J Med Genet 2002, 108:51-56.

9. Griseri P, Vos Y, Giorda R, Gimelli S, Beri S, Santamaria G, Mognato G, Hofstra RM, Gimelli G, Ceccherini I: Complex pathogenesis of Hirschsprung's disease in a patient with hydrocephalus, vesico-ureteral reflux and a balanced translocation $\mathrm{t}(3 ; 17)(\mathrm{p} 12 ; \mathrm{q} 11)$. Eur J Hum Genet 2009, 17:483-490.

10. Jackson SR, Guner YS, Woo R, Randolph LM, Ford H, Shin CE: L1CAM mutation in association with X-linked hydrocephalus and Hirschsprung's disease. Pediatr Surg Int 2009, 25:823-825.

11. Nakakimura S, Sasaki F, Okada T, Arisue A, Cho K, Yoshino M, Kanemura Y, Yamasaki M, Todo S: Hirschsprung's disease, acrocallosal syndrome, and congenital hydrocephalus: report of 2 patients and literature review. $J$ Pediatr Surg 2008, 43:E13-E17.

12. Schouten JP, McElgunn CJ, Waaijer R, Zwijnenburg D, Diepvens F, Pals G: Relative quantification of 40 nucleic acid sequences by multiplex ligation-dependent probe amplification. Nucleic Acids Res 2002, 30:e57.

13. Allen RC, Zoghbi HY, Moseley AB, Rosenblatt HM, Belmont JW: Methylation of Hpall and Hhal sites near the polymorphic CAG repeat in the human androgen-receptor gene correlates with $\mathrm{X}$ chromosome inactivation. Am J Hum Genet 1992, 51:1229-1239.

14. Ruiz-Ferrer F, Fernandez R, Antiñolo G, López-Alonso M, Eng C, Borrego S: A complex additive of inheritance for Hirschprung disease (HSCR) is supported by both RET mutations and predisposing RET haplotypes. Genet Med 2006, 8:1-6.

15. Sánchez-Mejías A, Fernández RM, López-Alonso M, Antiñolo G, Borrego S: New roles of EDNRB and EDN3 in the pathogenesis of Hirschsprung disease. Genet Med 2010, 12:39-43.

16. Fernández RM, Sánchez-Mejías A, Ruiz-Ferrer M, López-Alonso M, Antiñolo G, Borrego S: Is the RET proto-oncogene involved in the pathogenesis of intestinal neuronal dysplasia type B? Mol Med Rep 2009, 2:265-270.

17. Borrego S, Wright FA, Fernandez RM, Williams N, Lopez-Alonso M, Davuluri R, Antiñolo G, Eng C: A founding locus within the RET protooncogene may account for a large proportion of apparently sporadic Hirschsprung disease and a subset of cases of sporadic medullary thyroid carcinoma. Am J Hum Genet 2003, 72:88-100.

18. Fernandez RM, Boru G, Pecina A, Jones K, Lopez-Alonso M, Antiñolo G, Borrego S, Eng C: Ancestral RET haplotype associated with Hirschsprung's disease shows linkage disequilibrium breakpoint at -1249. J Med Genet 2005, 42:322-327.

19. del Gaudio D, Fang P, Scaglia F, Ward PA, Craigen WJ, Glaze DG, Neul JL, Patel A, Lee JA, Irons M, Berry SA, Pursley AA, Grebe TA, Freedenberg D, Martin RA, Hsich GE, Khera JR, Friedman NR, Zoghbi HY, Eng CM, Lupski JR, Beaudet AL, Cheung SW, Roa BB: Increased MECP2 gene copy number as the result of genomic duplication in neurodevelopmentally delayed males. Genet Med 2006, 8:784-792.

20. Van Esch $H$, Bauters $M$, Ignatius J, Jansen $M$, Raynaud $M$, Hollanders $K$, Lugtenberg D, Bienvenu T, Jensen LR, Gecz J, Moraine C, Marynen P, Fryns JP, Froyen G: Duplication of the MECP2 region is a frequent cause of severe mental retardation and progressive neurological symptoms in males. Am J Hum Genet 2005, 77:442-453.

21. Clayton-Smith J, Walters S, Hobson E, Burkitt-Wright E, Smith R, Toutain A, Amiel J, Lyonnet S, Mansour S, Fitzpatrick D, Ciccone R, Ricca I, Zuffardi O, Donnai D: Xq28 duplication presenting with intestinal and bladder dysfunction and a distinctive facial appearance. Eur J Hum Genet 2009, 17:434-443.

22. Gargiulo A, Auricchio R, Barone MV, Cotugno G, Reardon W, Milla PJ, Ballabio A, Ciccodicola A, Auricchio A: Filamin A is mutated in X-linked chronic idiopathic intestinal pseudo-obstruction with central nervous system involvement. Am J Hum Genet 2007, 80:751-758.

23. Heanue TA, Pachnis V: Enteric nervous system development and Hirschsprung's disease: advances in genetic and stem cell studies. Nat Rev Neurosci 2007, 8:466-479.

24. Moore SW: The contribution of associated congenital anomalies in understanding Hirschsprung's disease. Pediatr Surg Int 2006, 22:305-315.

25. Basel-Vanagaite L, Straussberg R, Friez MJ, Inbar D, Korenreich L, Shohat M, Schwartz CE: Expanding the phenotypic spectrum of L1CAM-associated disease. Clin Genet 2006, 69:414-419. 


\section{Pre-publication history}

The pre-publication history for this paper can be accessed here: http://www.biomedcentral.com/1471-2350/11/137/prepub

doi:10.1186/1471-2350-11-137

Cite this article as: Fernández et al:: Novel association of severe

neonatal encephalopathy and Hirschsprung disease in a male with a

duplication at the Xq28 region. BMC Medical Genetics 2010 11:137.

Submit your next manuscript to BioMed Central and take full advantage of:

- Convenient online submission

- Thorough peer review

- No space constraints or color figure charges

- Immediate publication on acceptance

- Inclusion in PubMed, CAS, Scopus and Google Scholar

- Research which is freely available for redistribution

Submit your manuscript at www.biomedcentral.com/submit 